\title{
IMAGE-BASED DETECTION OF CORPUS CALLOSUM VARIABILITY FOR MORE ACCURATE DISCRIMINATION BETWEEN AUTISTIC AND NORMAL BRAINS
}

\author{
Ahmed Elnakib ${ }^{1}$, Ayman El-Baz ${ }^{1 *}$, Manuel F. Casanova ${ }^{2}$, Georgy Gimel'farb ${ }^{3}$, and Andrew E. Switala ${ }^{2}$ \\ ${ }^{1}$ BioImaging Laboratory, Bioengineering Department, University of Louisville, Louisville, KY, USA. \\ 2 Department of Psychiatry and Behavioral Science, University of Louisville, Louisville, KY, USA. \\ 3 Department of Computer Science, University of Auckland, Auckland, New Zealand.
}

\begin{abstract}
The importance of accurate early diagnostics of autism that severely affects personal behavior and communication skills cannot be overstated. Neuropathological studies have revealed an abnormal anatomy of the Corpus Callosum (CC) in autistic brains. We propose a new approach to quantitative analysis of three-dimensional (3D) magnetic resonance images (MRI) of the brain that ensures a more accurate quantification of anatomical differences between the $\mathrm{CC}$ of autistic and normal subjects. It consists of three main processing steps: (i) segmenting the $\mathrm{CC}$ from a given 3D MRI using the learned CC shape and visual appearance; (ii) extracting a centerline of the $\mathrm{CC}$; and (iii) cylindrical mapping of the $\mathrm{CC}$ surface for its comparative analysis. Our experiments revealed significant differences (at the $95 \%$ confidence level) between 17 normal and 17 autistic subjects in four anatomical divisions, i.e. splenium, rostrum, genu and body of their CC.
\end{abstract}

Index Terms- Segmentation, Modeling, Corpus Callosum, Autism.

\section{INTRODUCTION}

Autistic Spectrum Disorder (ASD), or autism, is a complex neurological disability characterized by qualitative abnormalities in behavior and higher cognitive functions [1]. It typically appears during the first three years of life and impacts development of social interaction and communication skills. According to the Centers for Disease Control and Prevention (CDC), about 1 in 110 American children fall somewhere in the autistic spectrum. Although the cause of autism is still largely not clear, researchers have suggested that genetic, developmental, and environmental factors may be the cause or the predisposing effects towards developing autism [2]. Multiple studies during the past decade have revealed that different brain structures are involved in the abnormal neuro-development associated with autism. For example, MRI studies have shown an increased volume in cerebellar white matter of young children with autism relative to controls [3]. Also, cortical grey matter enlargement, particularly in the frontal and temporal lobes, is another abnormal feature of the brain in autistic patients [4]. This paper develops a new framework for analyzing the surface of CC for normal and autistic subjects. The goal is to identify whether or not the $\mathrm{CC}$ involved in the abnormal neural development is associated with autism.

The CC is the largest fiber bundle connecting the left and the right cerebral hemispheres in the human brain. Since the higher cognitive functions of the brain are highly affected by the impaired communication between the hemispheres, several studies [5-12] have proposed to analyze the $\mathrm{CC}$ for autistic subjects. In [5-8], the $\mathrm{CC}$ had been traced from the midsagittal MRI slice. Statistical difference analysis was applied to find out which part in the CC contributes significantly to identification of autistic brains. Chung et al. [9] applied a voxel based

\footnotetext{
* Corresponding Author:- Tel: (502) 852-5092, Fax: (502) 852-6806,
} E-mail: aselba01@louisville.edu morphometry approach using a freely available public domain software package (i.e., SPM99 software [13]) to spatially normalize the midsagital MRI slice to a common stereotactic space in order to segment the $\mathrm{CC}$ and localize the $\mathrm{CC}$ subregions that are related to autism. To cover more CC anatomy, studies [10-12] account not only for the midsagital slice but also for four adjacent slices on both sides. He et al., [10] traced the $\mathrm{CC}$ from the nine slices based on a semi automated active contour methodology. A contour stitching technique was applied to create the 3D CC surfaces for each subject. Statistical difference analysis was applied to the signed distance map from each subject surface to a template. Instead of using a signed distance map metric, Vidal et al., [12] utilized the CC thickness-the distance between uniformly spaced points on the $\mathrm{CC}$ surface to the $\mathrm{CC}$ medial line (i.e., the average curve between superior and inferior $\mathrm{CC}$ boundaries)- to localize regions of callosal thinning in autism.

To the best of our knowledge, all the previous works have focused on analyzing either the $2 \mathrm{D}$ cross section of the midsagittal of the $\mathrm{CC}$ or the midsagital slice along with four adjacent slices on both sides. Unfortunately, this is insufficient for detecting the whole anatomic variability of the $\mathrm{CC}$ of autistic subjects. To ensure a complete $3 \mathrm{D}$ analysis, the whole $\mathrm{CC}$ surface (traced from all the slices in which the CC appears) is mapped onto a cylinder in such a way as to compare more accurately various autistic and normal CC. Our cylindrical mapping has been inspired by the functional conformal mapping [14]. Similar to the conformal mapping, it is a bijective (one-to-one) transformation and preserves angular relationships between the points. For these reasons, the conformal mapping was recently considered an efficient technique for surface matching [15] and visualization of various anatomic structures [16].

The paper is organized as follows: Section 2 overviews in brief our CC segmentation using a learned soft CC shape model and an identifiable joint Markov-Gibbs random field (MGRF) model of 3D MRI and "object-background" region maps. Section 3 details the extraction of the centerline of the segmented CC by solving the Eikonal equation. The cylindrical mapping of the $\mathrm{CC}$ after finding its centerline is described in Section 4. Experimental results and conclusions are presented in Section 5.

\section{SEGMENTATION OF CORPUS CALLOSUM USING A SHAPE MODEL AND A JOINT MGRF MODEL OF 3D MRI}

Let $\mathbf{Q}=\{0, \ldots, Q-1\}, \mathbf{L}=\{\mathrm{ob}, \mathrm{bg}\}$, and $\mathbf{U}=[0,1]$ be a set of $Q$ integer gray levels, a set of object ("ob") and background ("bg") labels, and a unit interval, respectively. Let a 3D arithmetic grid $\mathbf{R}=\{(x, y, z): x=0,1, \ldots, X-1 ; y=0,1, \ldots, Y-$ $1 ; z=0,1, \ldots, Z-1\}$ support grayscale MRI $\mathbf{g}: \mathbf{R} \rightarrow \mathbf{Q}$, their binary region maps $\mathbf{m}: \mathbf{R} \rightarrow \mathbf{L}$, and probabilistic shape model $\mathbf{s}: \mathbf{R} \rightarrow \mathbf{U}$. The shape model allows for registering (aligning) 3D brain MRI. The co-registered 3D MRI and their region maps are modeled with a joint MGRF specified by a probability distribution $P(\mathbf{g}, \mathbf{s}, \mathbf{m})=P(\mathbf{g} \mid \mathbf{m}) \mathbf{P}(\mathbf{s} \mid \mathbf{m}) P(\mathbf{m})$ where $P(\mathbf{m})$ is an uncondi- 
tional Gibbs distribution of co-registered region maps, $P(\mathbf{g} \mid \mathbf{m})$ is a conditional distribution of the MRI signals given the map, and $P(\mathbf{s} \mid \mathbf{m})$ is a conditional distribution of the prior shape of the $\mathrm{CC}$ given the map.

We focus on accurate identification of spatial voxel interactions in $P(\mathbf{m})$, voxel-wise distributions of intensities in $P(\mathbf{g} \mid \mathbf{m})$, and prior distribution of the shape of the CC in $P(\mathbf{s} \mid \mathbf{m})$ for co-aligned 3D MR images. The probabilistic 3D shape model $\mathbf{s}$ is learned from a training set of manually segmented and co-aligned images. To perform the initial CC segmentation, every given MRI is aligned to one of the training images. The shape model provides the voxel-wise object and background probabilities being used, together with the conditional image intensity model $P(\mathbf{g} \mid \mathbf{m})$, to build an initial region map. The final Bayesian segmentation is performed using the identified joint MGRF model of the MRI and region maps.

\subsection{Spatial voxel interaction in the $\mathrm{CC}$}

A generic MGRF of region maps accounts only for pairwise interaction between each region label and its neighbors. Generally, the interaction structure and Gibbs potentials are arbitrary and can be identified from the training data. For simplicity, we restrict the interaction structure to the nearest voxels only, (i.e. to the voxel 26-neighborhood), and assume, by symmetry considerations, that the potentials depend only on intra- or inter-region position of each voxel pair (i.e. whether the labels are equal or not) but are independent of its relative orientation. Under these restrictions, it is similar to the conventional auto-binomial model and differs only in that the potentials are estimated analytically.

The 26-neighborhood has three types of symmetric pairwise interactions specified by the absolute distance $a$ between two voxels in the same and adjacent MRI slices $(a=1, \sqrt{2}$, and $\sqrt{3}$, respectively): (i) the closest pairs with the inter-voxel coordinate offsets $\mathbf{N}_{1}=\{(1,0,0),(0,1,0),(0,0,1)\}$; (ii) the farther diagonal pairs with the offsets $\mathbf{N}_{\sqrt{2}}=\{(0,1, \pm 1),(1,0, \pm 1),(1, \pm 1,0)\}$, and (iii) the farthest diagonal pairs with the offsets $\mathbf{N}_{\sqrt{3}}=\{(1, \pm 1, \pm 1)\}$. The potentials of each type are bi-valued because only the coincidence of the labels is taken into account: $\mathbf{V}_{a}=\left\{V_{a, \text { eq }} ; V_{a, \text { ne }}\right\}$ where $V_{a, \text { eq }}=V_{a}\left(l, l^{\prime}\right)$ if $l=l^{\prime}$ and $V_{a, \text { ne }}=V_{a}\left(l, l^{\prime}\right)$ if $l \neq l^{\prime} ; a \in \mathbf{A}=$ $\{1, \sqrt{2}, \sqrt{3}\}$. Then the MGRF model of region maps is as follows:

$P(\mathbf{m}) \propto \exp \sum_{(x, y, z) \in \mathbf{R}} \sum_{a \in \mathbf{A}} \sum_{(\xi, \eta, \kappa) \in \mathbf{N}_{a}} V_{a}\left(m_{x, y, z}, m_{x+\xi, y+\eta, z+\kappa}\right)$

To identify the MGRF model described in the equation, analytical maximum likelihood estimates are formed in line with [17] as follows:

$$
V_{a, \mathrm{eq}}=-V_{a, \mathrm{ne}}=4\left(f_{a, \mathrm{eq}}(\mathbf{m})-\frac{1}{2}\right)
$$

where $f_{a, \text { eq }}(\mathbf{m})$ denotes the relative frequency of the equal label pairs in the equivalent voxel pairs $\{((x, y, z),(x+\xi, y+\eta, z+\kappa))$ : $\left.(x, y, z) \in \mathbf{R} ;(x+\xi, y+\eta, z+\kappa) \in \mathbf{R} ;(\xi, \eta, \kappa) \in \mathbf{N}_{a}\right\}$.

\subsection{Conditional intensity model for the $3 D$ MRI}

We use a simple random field of conditionally independent intensities to model the 3D MRI, given a region map:

$$
P(\mathbf{g} \mid \mathbf{m})=\prod_{(x, y, z) \in \mathbf{R}} p_{m_{x, y, z}}\left(g_{x, y, z}\right)
$$

where the voxel-wise probability distributions for the $\mathrm{CC}$ and its background, $p_{\lambda}=\left[p_{\lambda}(q): q \in \mathbf{Q}\right] ; \lambda \in \mathbf{L}$, are estimated during the segmentation process. To separate $p_{\text {ob }}$ and $p_{\text {bg }}$, the mixed empirical distribution of all the voxel intensities is approximated with a linear combination of discrete Gaussians (LCDG) ${ }^{1}$.

\footnotetext{
${ }^{1}$ A discrete Gaussian (DG) $\Psi_{\theta}=(\psi(q \mid \theta): q \in \mathbf{Q})$ with $\theta=\left(\mu, \sigma^{2}\right)$ is defined [18] as $\psi(q \mid \theta)=\Phi_{\theta}(q+0.5)-\Phi_{\theta}(q-0.5)$ for $q=1, \ldots, Q-2$, $\psi(0 \mid \theta)=\Phi_{\theta}(0.5), \psi(Q-1 \mid \theta)=1-\Phi_{\theta}(Q-1.5)$ where $\Phi_{\theta}(q)$ is the cumulative Gaussian function with the mean $\mu$ and the variance $\sigma^{2}$.
}

In this case the LCDG has two dominant positive DGs that represent modes associated with the object (i.e. CC) and background, respectively, in the empirical intensity distribution for the MRI to be segmented. To approximate more closely this distribution, the LCDG also contains a number of positive and negative subordinate DGs:

$$
p_{\mathrm{LCDG}}(q)=\sum_{t=1}^{C_{\mathrm{p}}} w_{\mathrm{p}, t} \psi\left(q \mid \theta_{\mathrm{p}, t}\right)-\sum_{t=1}^{C_{\mathrm{n}}} w_{\mathrm{n}, t} \psi\left(q \mid \theta_{\mathrm{n}, t}\right)
$$

where the index $\alpha \in\{\mathrm{p}, \mathrm{n}\}$ specifies whether the DG is positive or negative, $C_{\alpha}$ is the number of such components, and $\theta_{\alpha, t}$ and $w_{\alpha, t}$ denote the weight and parameters of each individual DG $\Psi_{\theta_{\alpha, t}} ; t=$ $1, \ldots, c_{\alpha}$, respectively. The LCDG of Eq. (2), including the numbers $C_{\mathrm{p}}$ and $C_{\mathrm{n}}$ of its components, is identified using our previous EMbased algorithm introduced in [18].

\subsection{Probabilistic model of the $\mathrm{CC}$ shape}

Most of the recent works on image segmentation use level set based representations of shapes: an individual shape is outlined by a set of boundary pixels (or voxels) at the zero level of a certain distance function, and a given shape is approximated with the closest linear combination of the training shapes. The main drawback of this representation is that the space of signed distances is not closed with respect to linear operations. As a result, linear combinations of the distance functions may relate to invalid or even physically impossible boundaries.

To circumvent this limitation, the probabilistic 3D CC shape model $\mathbf{s}: \mathbf{R} \rightarrow \mathbf{U}$ where $s(x, y, z)$ is the empirical probability that the voxel $(x, y, z)$ belongs to the $\mathrm{CC}$ is learned from the co-registered training MRI. Such a prior is constructed by co-aligning the training set of MRI by a rigid 3D registration using mutual information as similarity measure [19], segmenting the CCs by hand from the aligned set, and counting how many times each voxel $s(x, y, z)$ was segmented as the $\mathrm{CC}$.

\subsection{Segmentation algorithm}

In total, the proposed $\mathrm{CC}$ segmentation is obtained by the following processing steps:

1. Perform an affine alignment of a given 3D MRI to an arbitrary prototype $\mathrm{CC}$ from the training set using mutual information as a similarity measure.

2. Estimate the conditional intensity model $P(\mathbf{g} \mid \mathbf{m})$ by identifying the bimodal LCDG.

3. Use the intensity model found and the learned probabilistic shape model to perform an initial segmentation of the CC, i.e. to form an initial region map.

4. Use the initial region map to identify the MGRF model $P(\mathbf{m})$ of region maps and update the conditional intensity model $P(\mathbf{g} \mid \mathbf{m})$.

5. Perform the final Bayesian segmentation of the $\mathrm{CC}$ in accord with the updated joint MGRF model $P(\mathbf{g}, \mathbf{m})$.

\section{CENTERLINE EXTRACTION FROM THE CC}

The problem of extracting the centerline connecting splenium (e.g. the point $A$ in Fig. 1(a)) with rostrum (the point $B$ ) can be formulated as a minimum-cost problem: find the path that minimizes the cumulative cost of traveling from the starting point $A$ to the destination $B$. As defined in [20], if $W(x, y, z)$ is a cost function at any location $(x, y, z)$ inside the $\mathrm{CC}$ then the minimum cumulative cost at the location $B=$ $\left(x^{\prime}, y^{\prime}, z^{\prime}\right)$ is

$$
T(B)=\min _{C_{A B}} \int_{0}^{L} W(C(l)) d l
$$


where $L$ is the path length and $C_{A B}$ is a set of all possible paths linking $A$ to $B$ such that $C(0)=A$ and $C(L)=B$ are the starting and ending points of each path $C(l) \in C_{A B}$. The minimum cost path solving Eq. (3) also satisfies the solution of the Eikonal equation:

$$
|\nabla T(x, y, z)| F(x, y, z)=1
$$

where $T(x, y, z)$ is the time at which the front evolving from the point $A$ crosses the point $(x, y, z)$, and $F(x, y, z)$ is the speed function.

We propose a new algorithm to extract the centerline of the $3 \mathrm{D} \mathrm{CC}$ based on solving Eq. (4):

1. Find the boundary of the segmented $\mathrm{CC}$ by estimating its $3 \mathrm{D}$ edges (see Fig. 1(b)).

2. Find the normalized minimum Euclidian distance $D(x, y, z)$ from every inner $\mathrm{CC}$ point $(x, y, z)$ to the $\mathrm{CC}$ boundary (Fig. 1(c)) by solving Eq. (4) using the fast marching level sets at the unit speed function, $F(x, y, z)=1$ [21].

3. Extract points located on the $3 \mathrm{D}$ centerline of the $\mathrm{CC}$ as follows:

(a) Pick any splenium point as a starting point, $A$.

(b) Propagate an orthogonal wave from the point $A$ by solving Eq. (4) using the fast marching level sets at the speed function $F(x, y, z)=\exp (-D(x, y, z))$ (Fig. 1(d)).

(c) Track the point with the maximum curvature for each propagating wave front $($ Fig. $1(\mathrm{e}, \mathrm{f}))$, this point being considered at any time as corresponding to the starting point A.

(d) The point $B$ at which the maximum curvature point of the propagating wave hits rostrum of the $\mathrm{CC}$ is selected as the end point of the centerline.
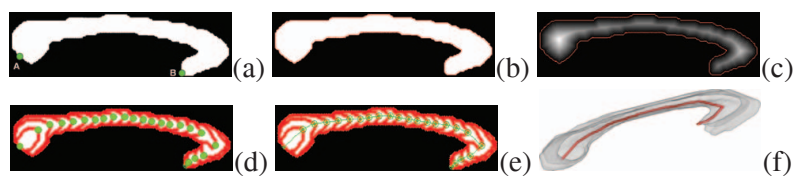

Fig. 1. Steps of the proposed centerline algorithm illustrated by the saggital 2D cross-sections of the 3D CC (a), estimated 3D CC edges (b), normalized distance map (c), orthogonal wave propagated from the point $A(\mathrm{~d})$, extracted centerline (e), and the 3D visualization of the extracted centerline (f).

\section{CYLINDRIC MAPPING TO EVALUATE CC VARIABILITY}

We reveal differences between the autistic and normal CC by using cylindric transformation. Before applying the cylindric transformation, the extracted $3 \mathrm{D} \mathrm{CC}$ is re-sliced by generating planes that are orthogonal to and equidistant along the centerline as shown in Fig. 2(a,b). The re-slicing transforms 3D coordinates $(x, y, z)$ of the voxels associated with each slice $k$ into specific new coordinates $(i, j, k)$ where $(i, j)$ are the $2 \mathrm{D}$ coordinates on the corresponding slicing plane $k$. A boundary point $(i, j)$ of each slice $k$ is related to the surface of a cylinder with a fixed radius $\rho$ as shown in Fig. 3. The rectified centerline of the $\mathrm{CC}$ is superposed onto the cylinder axis. Polar coordinates $(r, \theta)$ of the boundary point $(i, j, k)$ with respect to the slice center $\left(i_{0}, j_{0}, k\right)$, being the trace of the centerline:

$$
r=\sqrt{\left(i_{0}-i\right)^{2}+\left(j_{0}-j\right)^{2}} ; \quad \theta=\tan ^{-1}\left(\frac{j-j_{0}}{i-i_{0}}\right)
$$

associate the point $(i, j, k) \equiv(r, \theta, k)$ with the point $(\rho, \theta, k)$ on the cylindric surface. The resulting distribution of the radii $r$ over this surface represents the segmented CC.
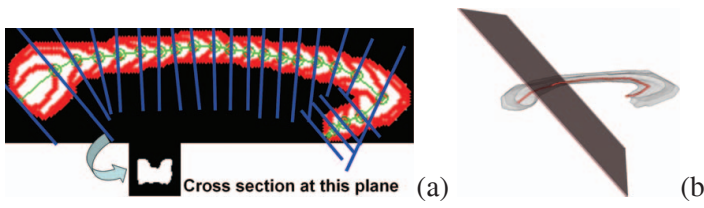

(b)

Fio. 2. 2D (a) and 3D (h) illustrations of re-slicino

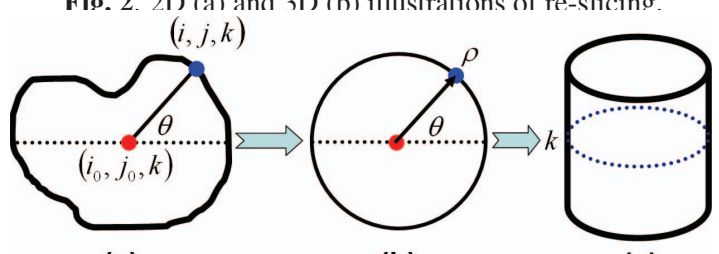

(a)

(b)

(c)

Fig. 3. The proposed cylindric mapping: a cross-section of the resliced CC (a), the CC cross-section mapped onto a circle (b), placing the circle onto the corresponding location in the cylinder (c).

\section{EXPERIMENTAL RESULTS AND CONCLUSIONS}

The proposed approach has been tested on in-vivo data collected from 17 autistic subjects aged 16 to 22 years, and a group of 17 controls who match for gender, age, educational level, socioeconomic background, handedness, and general intelligence. All the subjects are physically healthy and free of history of neurological diseases and head injury. Briefly, all the subjects have exactly the same psychiatric conditions. All images were acquired with the same 1.5T MRI scanner (GE, Milwaukee, Wisconsin) with voxel resolution $1.0 \times 1.0 \times 1.25 \mathrm{~mm}^{3}$ using a T1 weighted imaging sequence protocol. The "ground truth" diagnosis to evaluate the classification accuracy for each patient was given by clinicians.

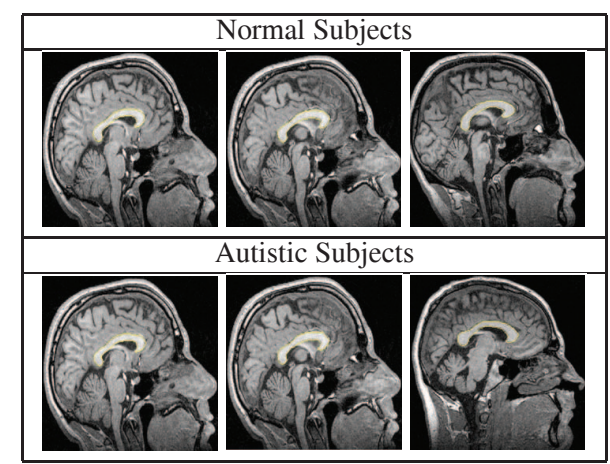

Fig. 4. 2D visualization of the segmented CC.

The results of the proposed CC segmentation algorithm are illustrated in Fig. 4, and Table 1 shows comparative results for the 15 data sets which are not used in the training with the known ground truth (manually segmented by an expert). The differences in the mean errors between the proposed segmentation, the level-set shape based approach of Tsai et al. [22], and ASM segmentation [23] are statistically significant according to the unpaired $t$-test (the two-tailed value $P$ is less than 0.0001).

Figures 5(a) and 5(b) present the average cylindrical maps for 17 normal subjects and 17 autistic subjects. As shown in Fig. 5(c) some locations in these maps differ significantly (at the 95\% confidence interval) for the normal and autistic subjects. The inverse cylindrical mapping outlines the significant areas on the average $\mathrm{CC}$ of normal subjects (see Fig. 6). These areas show that significant differences (at the $95 \%$ confidence interval) exist in the four anatomical divisions of 
Table 1. Accuracy of our segmentation on 15 data sets in comparison to the level sets based segmentation in [22] and the active shape model (ASM) in [23].

\begin{tabular}{|l|c|c|c|}
\cline { 2 - 4 } \multicolumn{1}{c|}{} & \multicolumn{3}{c|}{ Algorithm } \\
\cline { 2 - 4 } \multicolumn{1}{c|}{} & Our & {$[22]$} & {$[23]$} \\
\hline Minimum error, \% & $\mathbf{0 . 1 1}$ & 4.50 & 8.50 \\
Maximum error, \% & $\mathbf{1 . 8 7}$ & 11.8 & 19.10 \\
Mean error, \% & $\mathbf{0 . 9 7}$ & 5.11 & 10.13 \\
Standard deviation,\% & $\mathbf{1 . 0 7}$ & 3.10 & 9.10 \\
\hline Significant difference, P-value & & 0.0001 & 0.0001 \\
\hline
\end{tabular}

the CC, namely, in splenium, rostrum, genu, and body of the CC. Figure 6 demonstrates that the $\mathrm{CC}$ body for autistic subjects is thinner than for normal subjects.

In total, our preliminary results suggest that the proposed approach can detect significant differences in the four anatomical divisions of the $\mathrm{CC}$. These findings lead towards an efficient non-invasive computerassisted system for diagnosis of autism. In our future work, we aim at early diagnosis of autism by carrying out our procedure on young children and testing the potential of revealing their CCs' significant differences in detecting autism. Also, different brain structures will be investigated in order to quantitatively characterize the development and temporal changes of an autistic brain.
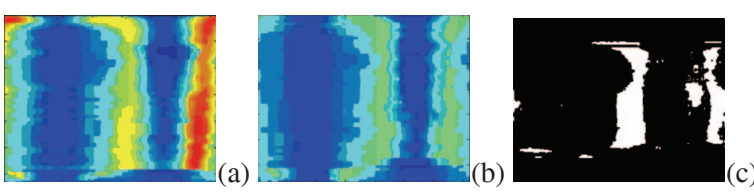

Fig. 5. Average cylindric maps of normal (a) and autistic (b) subjects and areas (c) of the $95 \%$-significant difference between normal and autistic subjects.

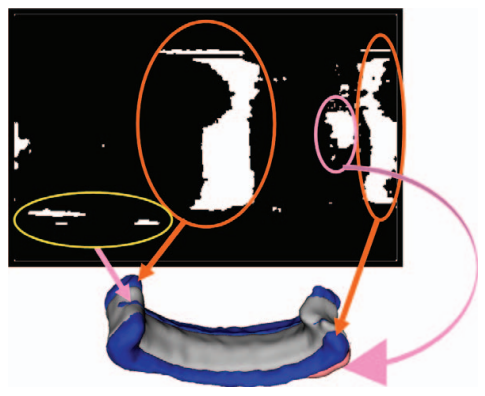

Fig. 6. Color-coded anatomical differences between the $\mathrm{CC}$ for normal and autistic subjects: the common parts (gray), parts that exist in normal and do not exist in autistic subjects (blue), and parts that exist in autistic and do not exist in normal subjects (pink)

\section{REFERENCES}

[1] P. Brambilla, A. Hardan, S. Nemi, "Brain anatomy and development in autism: review of MRI studies," Brain Research Bulletin, vol. 61, pp. 557-569, 2003.

[2] M. Stevens, D. Fein, M. Dunn, et al., "Subgroups of children with autism by cluster analysis: a longitudinal examination," $J$ Am Acad Child Adolesc Psychiatry, vol. 39, pp. 346-352, 2000.

[3] E. Courchesne et al., "nusual brain growth patterns in early life in patients with autistic disorder: an mri study," Neurology, vol. 57, no. 2, pp. 245-254, 2001.

[4] R. A. Carper, P. Moses, Z. D. Tigue, and E. Courchesne, "Cerebral lobes in autism: early hyperplasia and abnormal age effects," Neuroimage, vol. 16, pp. 1038-1051, 2002.
[5] B. Egaas, E. Courchesne, and O. Saitoh, "Reduced size of corpus callosum in autism," Arch. Neurology, vol. 52, no. 8, pp. 794801, 1995.

[6] J. Piven, J. Bailey, B.J. Ranson, and S. Arndt, "An MRI study of the corpus callosum in autism," Am. J. Psychiatry, vol. 154, no. 8, pp. 1051-1056, 1997.

[7] F. Manes, J. Piven, D. Vrancic, V. Nanclares, C. Plebst, and S. Starkstein, "An MRI study of the corpus callosum and cerebellum in mentally retarded autistic individuals," J. Neuropsychiatry Clin Neurosci, vol. 11, no. 4, pp. 470-474, 1999.

[8] A.Y. Hardan, N.J. Minshew, and M.S. Keshavan, "Corpus callosum size in autism," Neurology, vol. 55, pp. 1033-1036, 2000.

[9] M. K. Chung, K. M. Dalton, A. L. Alexander, and R. J. Davidson, "Less white matter concentration in autism: 2D voxel-based morphometry," Neuroimage, vol. 23, pp. 242-251, 2004.

[10] Q. He, Y. Duan, J. Miles, and N. Takahashi, "Statistical Shape Analysis of the CorpusCallosum in Subtypes of Autism," Proc. 7th IEEE Int. Conf. BIBE, 14-17 Oct. 2007, pp. 1087-1091.

[11] Q. He, K. Karsch, and Y. Duan, "Abnormalities in MRI traits of Corpus Callosum in Autism Subtype," Proc. 30th IEEE Int. Conf. EMBS, 20-25 Aug. 2008, pp. 3900-3903.

[12] C. N. Vidal, R. Nicolson, T. J. DeVito, K. M. Hayashi, J. A. Geaga, D.J. Drost, P. C. Williamson, N. Rajakumar, Y .Sui, R.A. Dutton, A. W. Toga, and P. M. Thompson, "Mapping corpus callosum deficits in autism: An index of aberrant cortical connectivity," Biol Psychiatry, vol. 60, no. 3,pp. 218-225, 2006.

[13] Statistical Parametric Mapping (SPM) Software, Available: http://www.fil.ion.ucl.ac.uk/spm/.

[14] R. Schinzinger, Conformal Mapping: Methods and Applications, Courier Dover Publications, 2003.

[15] S. Wang, Y. Wang, M. Jin, X. Gu, and D. Samaras, "Conformal geometry and its applications on 3D shape matching, recognition and stitching," IEEE Trans. Pattern Analysis and Machine Intelligence, vol. 29, no. 7, pp. 1029-1220, 2007.

[16] W. Hong, X.Gu, F. Qiu, M. Jin, and A. Kaufman, "Conformal virtual colon flattening," Proc. ACM Symp. Solid and Physical Modeling,Wales, UK, 2006, pp. 85-93.

[17] A. El-Baz and A. Farag, "Image Segmentation Using GMRF Models: Parameters Estimation and Applications," Proc. of IEEE International Conference on Image Processing (ICIP'03), Barcelona, Spain, September 1417, 2003, vol. II, pp. 177180.

[18] A. El-Baz and G. Gimel'farb, "EM Based Approximation of Empirical Distributions with Linear Combinations of Discrete Gaussians," Proc. IEEE Int. Conf. Image Processing, San Antonio, Texas, USA, Sept. 16-19, 2007, vol. 4, pp. 373-376.

[19] P. Viola and W. M. Wells, "Alignment by maximization of mutual information," Proc. 5th Int. Conf. Comp. Vision, 1995, pp. 16-23.

[20] L. Cohen and R. Kimmel, "Global minimum for active contour models: A minimal path approach," Int. J. Computer Vision, vol. 24, no. 1, pp. 57-78, 1997.

[21] D. Adalsteinsson and J. Sethian, "A fast level set method for propagating interfaces," J. Computational Physics, vol. 118, no. 2, pp. 269-277, 1995.

[22] A. Tsai, A. Yezzi, W. Wells, et al., "A shape based approach to the segmentation of medical imagery using level sets," IEEE Trans. Medical Imaging, vol. 22, pp. 137-154, 2003.

[23] T. Cootes and C. Taylor, "A mixture model for representing shape variation," Image Vision Computing, pp. 567-574, 1999. 